\title{
Unique lasing mechanism of localized dispersive nanostructures in InAs/InGaAlAs quantum dash broad interband laser
}

\author{
C. L. Tan ${ }^{\mathrm{a}}$, H. S. Djie ${ }^{\mathrm{b}}$, C. K. Tan ${ }^{\mathrm{c}}$, B. S. $\mathrm{Ooi}^{\mathrm{a}+}$ \\ ${ }^{a}$ Center for Optical Technologies and Department of Electrical and Computer Engineering, \\ Lehigh University, 7 Asa Drive, Bethlehem, PA, USA 18015; \\ ${ }^{\mathrm{b} J D S}$ Uniphase Corporation, San Jose, CA, USA 95134; \\ ${ }^{c}$ Department of Electronic and Electrical Eng., University of Sheffield, Sheffield S13JD, UK; \\ ${ }^{+}$Also with Division of Physical Sciences and Engineering, King Abdullah University of Science \\ and Technology (KAUST), Thuwal, Saudi Arabia \\ †Corresponding Author: bsooi@ieee.org
}

\begin{abstract}
The authors report on the nanowires-like and nanodots-like lasing behaviors in addition to multiple-wavelength interband transitions from InAs/InAlGaAs quantum dash (Qdash) lasers in the range of $\sim 1550 \mathrm{~nm}$. The presence of lasing actions simultaneously from two different dash ensembles, after postgrowth intermixing for crystalline quality improvement, indicate the absence of optical phonon emission due to the small variation in quantized interband transition energies. This effect is reproducible and shows different lasing characteristics from its quantum dot and quantum wire laser counterparts. Furthermore, the small energy spacing of only $25 \mathrm{~nm}$ (at center lasing wavelength of $\sim 1550 \mathrm{~nm}$ ) and the subsequent quenching of higher energy transition states at higher bias level in Qdash lasers suggest the absence of excited-state transition in highly inhomogeneous self-assembled Qdash structures. However, the appearance of a second lasing line in a certain range of high injection level, which is due to the presence of different sizes of dash assembles, corresponds to the transition from smaller size of Qdash ensembles in different planar active medium. This unique transition mechanism will affect the carrier dynamics, relaxation process in particular and further indicates localized finite carrier lifetime in all sizes of Qdash ensembles. These phenomena will lead to important consequences for the ground-state lasing efficiency and frequency modulation response of Qdash devices. In addition, these imply that proper manipulation of the Qdash ensembles will potentially result in localized nanolasers from individual ensemble and thus contributing towards enormously large envelope lasing coverage from semiconductor devices.
\end{abstract}

Keywords: Quantum-dash, broadband laser diode, quasi-supercontinuum, self-assembly, optical C-band wavelength, broad gain, postgrowth intermixing.

\section{INTRODUCTION}

Study of self-assembled semiconductor quantum islands in the shape of finite size wires (strongly elongated dots) called quantum-dashes (Qdashes) offers very promising properties for optoelectronic applications, especially in telecommunication lasers operating at $1.55 \mu \mathrm{m}$ and longer wavelengths [1-3]. Furthermore, it has been proven that the emission from Qdashes strongly depends on their sizes and can be tuned to any wavelength from 1.5 to $2 \mu \mathrm{m}$ by a simple change of the growth parameters [3,4]. Due to the size distribution, Qdash structures also offer a broad gain profile and therefore a wide emission bandwidth [5]. At a wavelength of $1.5 \mu \mathrm{m}$, a gain bandwidth of about $150 \mathrm{~nm}$ could be demonstrated, which is about three times larger than in conventional quantum-well (QW) lasers. However, up to now, there has been little detailed analysis reported on the consequences of dash formation on the electronic structure, which results in significantly different novel properties than the more commonly studied QW and selfassembled quantum-dot structures, especially the ultrabroad stimulated emission from highly inhomogeneous Qdashes $[1,5]$. Hereby, there is presented an optical study of InP-substrate-based structures with InAs Qdashes of three different height profiles, including the diffusion of electronic structure and multiple peak emission properties after postgrowth interdisffusion process, i.e. impurity-free vacancy disordering (IFVD) technique.

Novel In-Plane Semiconductor Lasers IX, edited by Alexey A. Belyanin, Peter M. Smowton,

Proc. of SPIE Vol. 7616, 761602 - (c) 2010 SPIE · CCC code: 0277-786X/10/\$18 · doi: 10.1117/12.842278 
In the context of stimulated emission properties from these highly inhomogeneous Qdash structures, a specified epitaxial growth parameter is chosen to demonstrate the widening profile of lasing envelope, which is crucial as a broadband light source. The advent of wavelength division multiplexing (WDM) networks has made the need for multi-wavelength and widely tunable laser source very compelling. Distributed feedback (DFB) lasers have been extensively used as sources for single-channel communications, and therefore, as a natural extension, WDM sources were built using an array of DFB lasers [6,7]. However, as channel spacing in the WDM systems continue to decrease for accommodating the bandwidth demand, the constraints on wavelength drifts of the laser sources become tighter. It is generally believed that a single multi-wavelength source or comb laser will be more robust to wavelength drifts than an array of single-wavelength sources [7, 8]. Furthermore, broad emission band lasers are highly desirable for sensing and low coherence imaging applications such as fiber gyroscopes, optical time domain reflectometry, sensing of various chemical agents, high-precision optical metrology and spectroscopy, and optical coherence tomography [9-11]. Multi-wavelength lasers operating in the $1500-\mathrm{nm}$ region have been developed using different gain mechanisms and media such as erbium-doped fiber amplifiers, semiconductor optical amplifier, and schemes based on stimulated Raman scattering gain and stimulated Brillouin scattering gain. In addition, hybrid gain mechanisms using a combination of the above mechanisms have also been used [7]. Compact broadband semiconductor lasers based on the intersubband quantum cascade principle have also been demonstrated in the mid-IR regime. However, these devices can only operate effectively under cryogenic temperatures; while suffer a dramatic reduction in the laser linewidth and the wall-plug efficiency resulted from high temperature (i.e. room temperature) operation [12]. Therefore, present advances in self-assembly semiconductor Qdash growth technology that permitted the fabrication of broadband laser utilizing more-efficient interband transition at room temperature become important.

In this paper, we demonstrate an experimental analysis of three InAs Qdashes broadband partial laser structures by utilizing IFVD technique at different degrees of intermixing, which basically involves process of dielectric cap annealing at different temperatures and time intervals. The observation of multiple equally-separated peaks emission in the Qdash structures from photoluminescence (PL) spectra and the corresponding Gaussian peak deconvolution suggests the presence of multiple quantized energy states. Moreover, the result of digital transitions among different quantized electronic states at higher degree of intermixing in the absence of quantized energy states local diffusion discards the prominent postulations of multiple excited states or biexciton emission [13] but the coexistence of many dash families possessing different crystalline quality. Based on the critical epitaxial growth parameter study, a full InAs/InAlGaAs Qdash laser structure is grown and further fabricated into devices. The characterization results show that the designed Qdash laser is capable of emitting a broadband spectrum over C-band at a large range of injection utilizing the cavity-dependent gain characteristics and the presence of multiple Qdash families across the stacking layers.

\section{EXPERIMENTS}

Three Qdash partial laser structures were grown using a gas source molecular beam epitaxy on (100) oriented n-doped InP substrates. The active region consists of four sheets of 5 monolayer InAs dashes, each embedded within a $7.6 \mathrm{~nm}$ thick compressively strained $\operatorname{In}_{0.64} \mathrm{Ga}_{0.16} \mathrm{Al}_{0.2}$ As quantum well [4] and varied thickness $(10 \mathrm{~nm}, 30 \mathrm{~nm}$ and $50 \mathrm{~nm}$ ) of tensile strained $\mathrm{In}_{0.50} \mathrm{Ga}_{0.32} \mathrm{Al}_{0.18} \mathrm{As}$ barrier, which are shown in fig. 1 (I) to (III), respectively. The reason behind the broadband lasing emission at room temperature resulted from these Qdash structures [4] were studied using low temperature (77K) PL and atomic force microscopy (AFM), utilizing surface Qdash partial laser structures. The active transition states in Qdash are dominantly affected by the dash height in growth direction as compared to other dimensions [4]. Hence, the morphology of the surface Qdash becomes a critical parameter and was investigated using a Veeco's Digital Instruments Dimension AFM system in ambient conditions and tapping mode. A fresh $200 \mathrm{~nm}$ layer of $\mathrm{SiO}_{2}$ was subsequently deposited on all samples using plasma enhanced chemical vapor deposition (PECVD). The IFVD process is then performed by annealing the $\mathrm{SiO}_{2}$ capped samples at four different temperatures $\left(700^{\circ} \mathrm{C}, 725^{\circ} \mathrm{C}\right.$, $\left.750^{\circ} \mathrm{C}, 775^{\circ} \mathrm{C}\right)$ in nitrogen ambient for four different time intervals $(30 \mathrm{~s}, 60 \mathrm{~s}, 90 \mathrm{~s}, 120 \mathrm{~s})$ in a rapid thermal processor. During the annealing, the porous $\mathrm{SiO}_{2}$ cap will enhance preferential atomic outdiffusion and therefore results in effective bandgap modification of Qdash. PL spectroscopy was then performed at $77 \mathrm{~K}$ using a $980 \mathrm{~nm}$ diode laser as an excitation source on the $\mathrm{SiO}_{2}$ capped samples. 


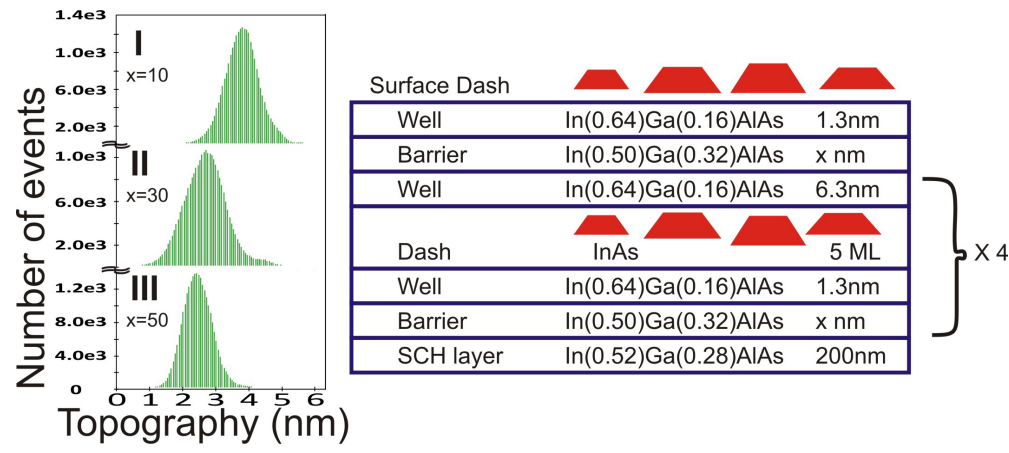

Fig. 1. Topography studies of surface InAs quantum dash with varying barrier width ( $\mathrm{x}$ of sample I - $10 \mathrm{~nm}$; II - $30 \mathrm{~nm}$; and III - $50 \mathrm{~nm}$ ) by using an atomic force microscopy system under tapping mode. The corresponding partial epitaxial structures are shown on the right.

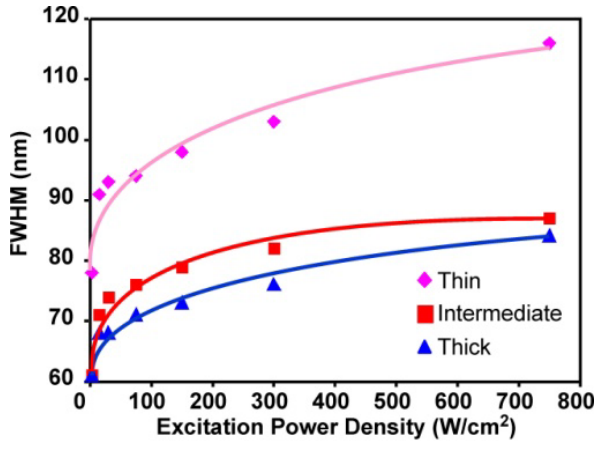

Fig. 2. The FWHM of PL emission from the 3 samples at different excitation power density. Sample with thin barrier is noted to exhibit enormously large PL bandwidth.

Based on the reported optimum intermixing process to obtain broadest emission envelope from Qdash lasers [10], we performed the dielectric cap annealing technique to induce selective intermixing using $475 \mathrm{~nm}$ thick $\mathrm{SiO}_{2}$ layer as a group-III vacancy source. The IFVD process to induce intermediate intermixing of Qdash nanostructures is performed by annealing the $\mathrm{SiO}_{2}$ capped sample at $750^{\circ} \mathrm{C}$ in nitrogen ambient for one minute in a rapid thermal processor. A full Qdash laser structure [10] with barrier width of $15 \mathrm{~nm}$ is used in the device fabrication. In order to maximize the gain [14], the optical cavity of the laser is aligned along the [011] orientation and is perpendicular to the dash direction. Standard broad area lasers with a $50 \mu \mathrm{m}$ wide oxide stripe were then fabricated from the intermixed samples. The lasers were cleaved into bars with different cavity lengths and tested on the temperature-controlled heat sink under pulsed operation ( $2 \mu$ s pulse width and $0.2 \%$ duty cycle). No facet coating was applied. Unless otherwise stated, the lasing spectra were acquired with a spectrum analyzer with a spectral resolution of $0.05 \mathrm{~nm}$.

\section{RESULTS AND DISCUSSION}

\subsection{Optical properties of Qdash material}

The topography of the three samples are depicted in fig 1 (I) to (III), respectively, corresponding to the partial structure dimension on the right. The reduced barrier thickness leads to an increased vertical strain coupling [15], which results in redshift of peak height of InAs Qdash as shown in fig. 1. The average dash height grown with thinnest barrier width $(3.87 \mathrm{~nm})$ is larger than intermediate $(2.78 \mathrm{~nm})$ and thickest $(2.53 \mathrm{~nm})$ barrier samples. An interesting result is the broadened dash height variation from the intermediate thick barrier sample, which has the largest envelope covering dash height dimension from the other 2 samples, i.e. full-width-half-maximum (FWHM) of $1.33 \mathrm{~nm}$ as compared to others with $\sim 1 \mathrm{~nm}$. This leads to an expected broadest PL linewidth from this particular sample due to the nonequilibrium distribution among largely inhomogeneous Qdash ensembles [16]. However, the results of PL measurements in fig. 2 suggest otherwise. The PL bandwidth of sample I with thinnest barrier width (FWHM of 116 $\mathrm{nm}$ at an excitation density of $750 \mathrm{~W} / \mathrm{cm}^{2}$ ) is much larger than the others (similar FWHM $\sim 85 \mathrm{~nm}$ at excitation power density of $750 \mathrm{~W} / \mathrm{cm}^{2}$ ) at all excitation. This phenomenon can be attributed to larger degree of dash height variation across different layers of Qdash. In other words, the effect of Qdash inhomogeneity on a single plane is negligible in broadening the PL emission resulted from multi localized active transition states.

The degree of dash height variation can be further studied via the PL peak shift with excitation density [not shown here]. The thickest Qdash ensembles in growth direction contribute to the largest degree of redshift of PL peak followed by samples with intermediate and thinnest Qdash ensembles. However, the degree of blueshift in a particular sample with increasing excitation power, which is due to the presence of localized noncoupling transition states from varied dimension of Qdash ensembles, illustrates the picture of morphology of Qdash ensembles. The largest degree of blueshift in sample I $(77 \mathrm{~nm})$ followed by thick $(66 \mathrm{~nm})$ and intermediate $(51 \mathrm{~nm})$ samples further proves that in-plane inhomogeneity is not significant as compared to dash height variation across the stacked layers of active materials. 


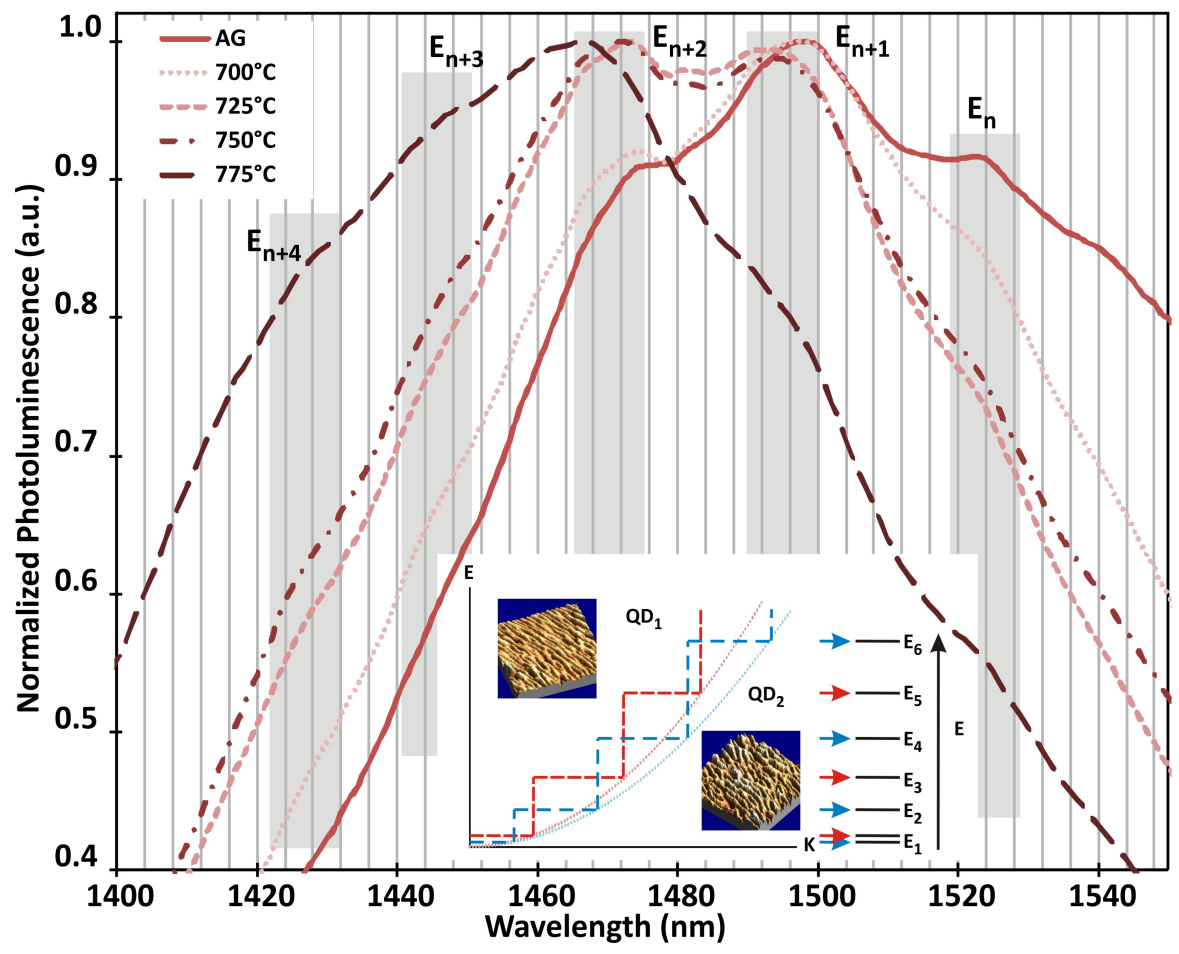

Fig. 3. 77K photoluminescence from the quantum dash structures (sample I) shows the intensity changes of multiple peak emission with different degrees of postgrowth bandgap engineering (annealing temperature). The irregular observation is originated from the wide distribution of dash height profiles, as depicted in the inset.

Hence, the broadband emission in both PL and electroluminescence measurements $[5,9,10]$ are only possible in the presence of stacked layers of Qdash active regions.

A detailed study of Qdash sample I after four different annealing temperatures for 30s of annealing time reveals many interesting results, as depicted in fig. 3. The $77 \mathrm{~K}$ PL measurements at $1000 \mathrm{~W} / \mathrm{cm}^{2}$ of excitation source show almost equally-spaced $(\sim 25 \mathrm{~nm})$ multiple emission humps, which indicates the presence of at least five different quantized energy levels in the Qdash system after a deconvolution study, as illustrated in fig. 4. The as-grown Qdash structure shows three major emission humps at $\sim 1520 \mathrm{~nm}, \sim 1495 \mathrm{~nm}$ and $\sim 1470 \mathrm{~nm}$, where each emission group possesses similar inhomogeneity broadening of $\sim 20 \mathrm{meV}$. At higher degree of intermixing in this experiment (i.e. highest annealing temperature at $775{ }^{\circ} \mathrm{C}$ ), the original three emission humps persist while two additional major emission humps at $\sim 1450 \mathrm{~nm}$ and $\sim 1425 \mathrm{~nm}$ are observed. From the deconvolution spectra in the intermixed sample [not shown here], the major emission humps are blue-shifted to smaller Qdash ensembles emitting at shorter wavelength. These results contradict with conventional observations and understanding of postgrowth interdiffusion, where each quantized energy states will diffuse in accordance to the degree of intermixing. Each ground state and excited state emission line from InP-based Qdash ensembles is expected to blue-shift with IFVD technique. However, the dwelling of all quantized energy states without diffusion with all degrees of intermixing in sample I suggest otherwise. The multiple almost equally spaced energy states could be attributed to the ground state emission from different Qdash families across the stacking layers of active region. Moreover, a mere transition of major PL peak intensity with intermixing postulates a digital transition among these Qdash ensembles of different crystalline qualities. As annealing temperature increases, the larger size of Qdash ensembles may be partly fragmentized into smaller Qdash ensembles and thus increase the population of Qdash families emitting at shorter wavelength. Furthermore, enhancement of short wavelength emission humps without the absence of longer wavelength humps at longer annealing time [not shown here] further supports the presence of multiple dash size families across the stacking layers and digital fragmentation of large-size Qdash ensembles. This postulation is further supported by the equal-spaced quantized energy levels, which is only possible in a system with multiple co-existing Qdashes having different energy-momentum properties, as illustrated in the inset of fig. 3. The stair-case profile of quantum-well energy density of states (DOS) is shown here instead of Qdash DOS for simplicity as a reference to the illustrated idea. 


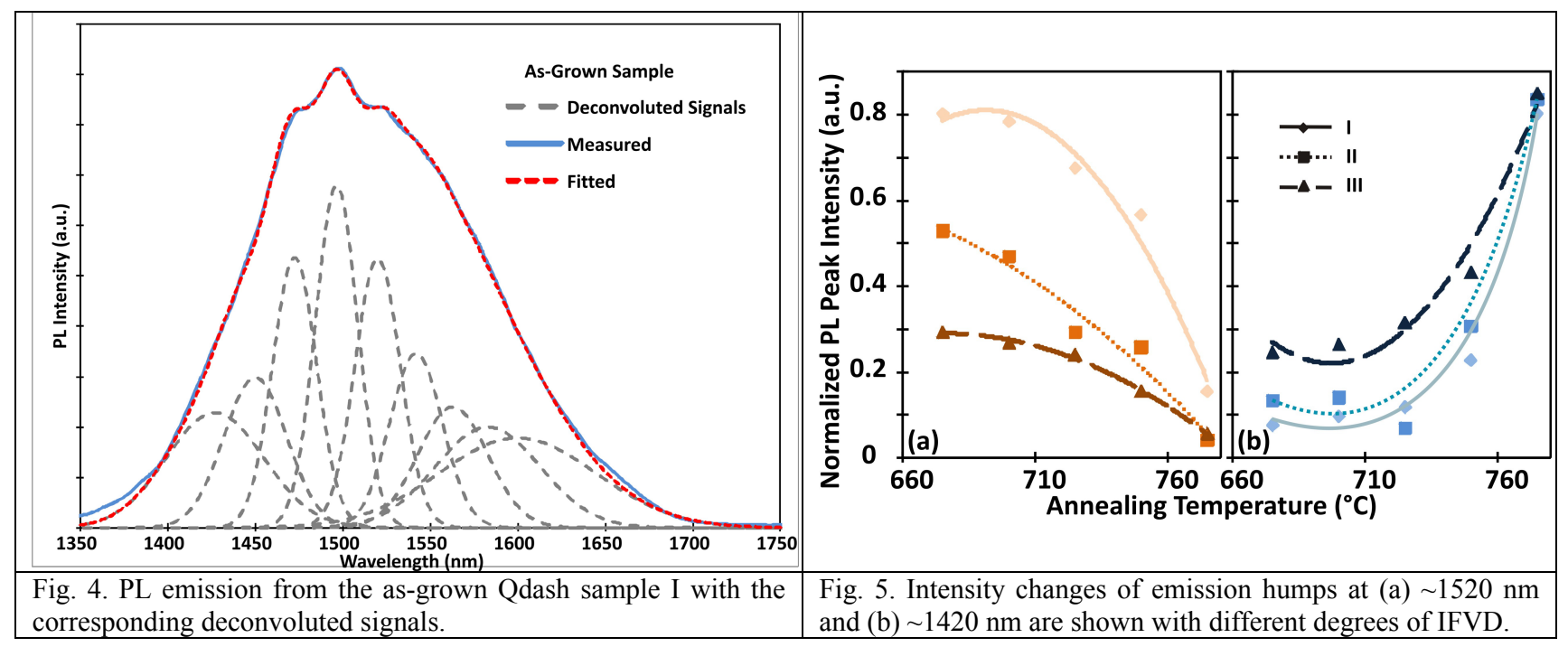

Similar experimental results are observed as well in both Qdash samples II and III. The PL intensity change of emission humps at long wavelength $(\sim 1520 \mathrm{~nm})$ and short wavelength $(\sim 1420 \mathrm{~nm})$ from three different samples at specified annealing temperatures are summarized in fig. 5. The results show that a thick Qdash system (height of $\sim 4$ $\mathrm{nm}$ ) is not stable and subjected to larger degree of emission intensity changes as compared to the other samples with higher degree of Qdash homogeneity. The comparatively big dash ensembles are prone to be fragmentized into smaller dash sizes. Hence, higher populations of smaller size Qdash will result with increasing annealing temperature and thereby peak PL intensity will be blue-shifted.

\subsection{Broad area laser characterization}

The devices were tested at different cavity lengths to evaluate the physical mechanism that drives the peculiar broadband characteristics resulted from a direct interband transition $[9,10]$. The lasing spectrum at constant injection level $\left(1.1 \mathrm{I}_{\mathrm{th}}\right)$ strongly relies on the device length, as shown in Fig. 6 . At $\mathrm{L}=800 \mu \mathrm{m}$ and shorter, the laser emission originates from a higher transition energy at $\sim 1525 \mathrm{~nm}$. For an intermediate long cavity (i.e. $\mathrm{L}=900 \mu \mathrm{m}$ and $\mathrm{L}=1000$ $\mu \mathrm{m})$ the carriers will fill up larger size dash ensembles due to the lower gain of the system and non-singularity of DOS in dimensionally long Qdash nanostructures, resulting in a center wavelength at $\sim 1560 \mathrm{~nm}$. The disparity of $\sim 35 \mathrm{~nm}$ between higher and lower transition may suggests the existence of different quantized energy in Qdash ensembles (i.e. the presence of many ground state levels originated from different Qdash ensembles). For short devices, the gain of the ground-state transition from big Qdash ensembles could be too small to compensate for the total loss, and lasing proceeds via the ground-state transition of smaller Qdash ensembles. However, at longer cavity (i.e. $\mathrm{L}=1200 \mu \mathrm{m}$ and above) the lasing peak blueshift to a center wavelength, which is identical to the transition energy observed in short cavity devices. This observation is contrary to that reported in both quantum dot (Qdot) [17] and quantum wire (Qwire) [18] lasers and thus validates the absence of excited-state transition in these highly inhomogeneous Qdash structures.

In Qdot/Qwire devices, the lasing wavelength at threshold is well known to be heavily dependent on the cavity length $[9,25,26]$. The localized carrier concentration in smaller size but high density dash ensembles will shows emission before others in short cavity devices, owing to the inherent high modal loss of the structure. At intermediate lengths, lasing proceed via larger size dash ensembles, which is similar to that reported in Qdot structures [19]. The progressive blueshift of lasing peak in longer cavity devices may be attributed to the nonequilibrium carrier distribution among different sizes of dash ensembles [16] and the existence of both dot and dash ensembles in a single active planar layer. As injection increases before reaching threshold condition in these long devices, carriers are expected to populate all dash ensembles regardless of size and shape. Lasing peak with center wavelength of $>1560 \mathrm{~nm}$ as compared to those from intermediate-long cavity devices is expected. However, larger amount of carriers are required to fulfill the population inversion requirement in larger size dash ensembles. Hence, carriers originally populated in the approximately big sizes of dash ensembles will shift to the larger one, owing to the weak $3 \mathrm{D}$ confinement for finite 


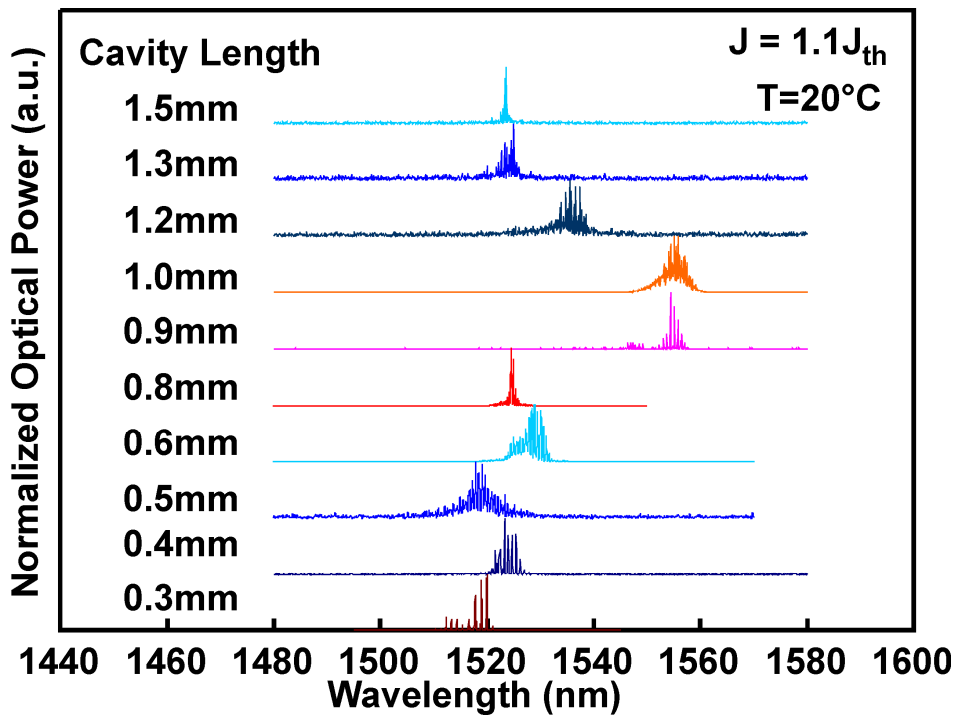

Fig. 6. Laser spectra measured under a current density of $1.1 \mathrm{Ith}$ at $20^{\circ} \mathrm{C}$ for InAs Qdash lasers with varying cavity lengths. The change of transitions with cavity lengths indicates racing conditions between different sizes of Qdash ensembles in achieving population inversion.

length dashes of $>30 \mathrm{~nm}$ [20]. Carrier population is preserved in a Qdot-like dash ensemble with dashes lengths comparable to the exciton Bohr diameter [21]. As injection keep increasing, population inversion will occur in smaller dash ensembles or Qdot-like dash ensembles before the lasing actions in larger dash ensembles. These observations suggest that broadband emission is likely to occur during the intermediate change of transition. In other words, both slightly shorter and longer cavity devices as compared to intermediate cavity length devices will emit a broadband emission. A more controllable method in achieving the broadband emission will be the utilization of facet coating, which will directly tailor the cavity loss as compared to the preference in device cavity length selection.

Broad area laser characterization from both the $900 \mu \mathrm{m}$ and $1500 \mu \mathrm{m}$ cavity-length Qdash devices further provides evidence of a multi-dashes emission as shown in fig. 7 (a) and (b), respectively. In $900 \mu \mathrm{m}$ long device, the appearance of higher-energy lasing line at slightly higher bias above threshold does not show saturated intensity that is twice the ground-state (lower-energy lasing line), which is corresponding to the double degeneracy of the excited-state in typical Qdot lasers [17]. Moreover, the integrated intensity of the ground-state transition neither saturates nor becomes increasingly suppressed with increasing injection, as observed in Qdot lasers [17]. In contrary, the highenergy lasing line is saturated and suppressed when injection increases. Furthermore, the small transition separation of $\sim 15 \mathrm{~nm}$ between the high-energy and low-energy lines shows less likelihood of two-state lasing from the same dot/dash family, corresponding to a dash geometry with the highest density. Hence, the two lasing lines observed in the Qdash structure suggest the presence of two dash ensembles with similar density, which is higher than others. This result agrees with the postulation suggested from previous Qdash partial laser structures studies. This phenomenon is different from that observed in both Qdot [17] and Qwire [18] lasers, suggesting that the combination of dot and wire structures on a same planar will introduce a new interesting lasing characteristic. Equally high density of dash ensembles will act as nanolasers, similar to that observed in nanowire lasers except the nature of randomness in selfassembled Qdash structure. In addition, the small energy difference between the two lasing dash ensembles $(\sim 8 \mathrm{meV})$, as compared to optical phonon energy in this material system of $\sim 34 \mathrm{meV}$, will hinder the emission of optical phonon, which is the dominant scattering process [22]. This contributes to the finite carrier lifetime in all dash ensembles and thus probability of population inversion increases in higher density dash ensembles. However, absorption of the shorter-wavelength light is inevitable in a same pumping region [22], thus causing the suppression of second lasing line when injection increases. In $1500 \mu \mathrm{m}$ long device, the lasing peak is at $\sim 1525 \mathrm{~nm}$ and shows continuous redshift with injection and the absence of second higher energy transition. Lower-energy line lasing becomes dominant with suppression of high-energy transition when injection is increased further, as shown in Fig. 7 (b). This may be attributed to the photon reabsorption in larger size of dash ensembles due to the physically same-planar of Qdashes with different geometries. 


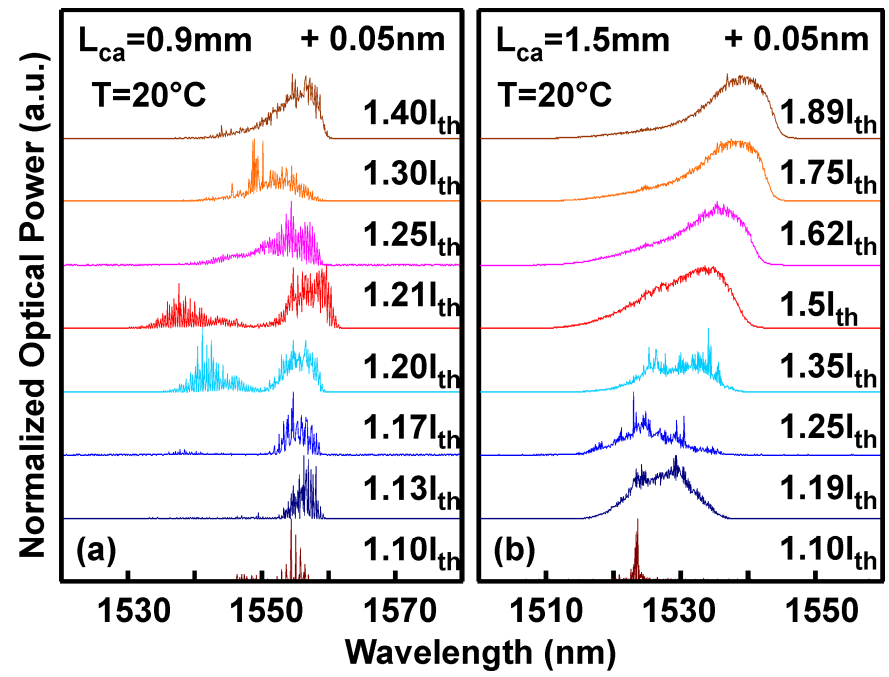

Fig. 7. The lasing spectra of (a) $900 \mu \mathrm{m}$ and (b) $1500 \mu \mathrm{m}$ cavity length device show the changes of lasing transitions from different groups of dash ensembles under increasing current injection. The lasing spectra above threshold condition at room temperature are acquired by an optical spectrum analyzer with wavelength resolution of $0.05 \mathrm{~nm}$.

\section{CONCLUSION}

In conclusion, the studies of Qdash partial laser structures show that planar Qdash inhomogeneity is not a critical parameter in realizing broadband lasing emission but dash height variation across multiple stacked active layers. A thin barrier width of $\sim 10 \mathrm{~nm}$ or less is necessary in an epitaxial growth layer of Qdash materials to enable quasisupercontinuum emission in semiconducting compounds. Moreover, the absence of diffusion in transition energy states but peak intensity change with postgrowth lattice disordering technique suggests the origin of broadband lasing action that is due to the presence of multiple Qdash ensembles of different sizes and crystalline qualities across the stacking layers. The fabricated devices exhibit different lasing properties with cavity length as compared to Qdot and Qwire counterparts. The wide spectral width lasing from the Qdash laser is attributed to the simultaneous lasing action from different sizes of Qdash ensembles, which is a preferable property over Qdot and Qwire systems for broadband laser realization. With proper wafer structure and growth optimizations, this new class of interband broadband laser can find various applications in sensing, optical communication in particular, imaging and diagnostic systems. Sufficiently long wavelengths also make such lasers compatible with optical and optoelectronic components based on silica fiber or silicon-based planar waveguides.

\section{ACKNOWLEDGEMENT}

This work is supported by National Science Foundation (Grant No. 0725647), US Army Research Laboratory, Commonwealth of Pennsylvania, Department of Community and Economic Development. Authors also acknowledge IQE Inc. for the growth of Qdash material.

\section{REFERENCES}

[1] S. C. Heck, S. Osborne, S. B. Healy, E. P. O’Reilly, F. Lelarge, F. Poingt, O. L. Gouezigou, and A. Accard, "Experimental and theoretical study of InAs/InGaAsP/InP quantum dah lasers", IEEE J. Quantum Electron. 45, 1508-1516 (2009).

[2] F. Lelarge, B. Dagens, J. Renaudier, R. Brenot, A. Accard, F. V. Dijk, D. Make, O. L. Gouezigou, J. -G. Provost, F. Poingt, J. Landreau, O. Drisse, E. Derouin, B. Rousseau, F. Pommereau, and G. -H. Duan, "Recent advances on 
InAs/InP quantum dash based semiconductor lasers and optical amplifiers operating at $1.55 \mu \mathrm{m}$, , IEEE J. Sel. Top. Quantum Electron. 13, 111-124, (2007).

[3] W. R-. Rudzinski, R. Kudrawiec, P. Podemski, G. Sek, J. Misiewicz, A. Somers, R. Schwertberger, J. P. Reithmaier, and A. Forchel, "Photoreflectance-probed excited states in InAs/InGaAlAs quantum dashes grown on InP substrate", Appl. Phys. Lett. 89, 031908 (2006).

[4] P. Podemski, G. Sek, K. Ryczko, J. Misiewicz, S. Hein, S. Hofling, A. Forchel, and G. Patriarche, "Columnar quantum dashes for an active region in polarization independent semiconductor optical amplifiers at $1.55 \mu \mathrm{m}$ ", Appl. Phys. Lett. 93, 171910 (2008).

[5] H. S. Djie, C. L. Tan, B. S. Ooi, J. C. M. Hwang, X. -M. Fang, Y. Wu, J. M. Fastenau, W. K. Liu, G. T. Dang, and W. H. Chang, "Ultrabroad stimulated emission from quantum-dash laser", Appl. Phys. Lett. 91, 111116 1-3, (2007).

[6] M. G. Young, U. Koren, and B. I. Miller, et al., "A 16x1 wavelength division multiplexer with integrated distributed Bragg reflector lasers and electroabsorption modulators", IEEE Photon. Technol. Lett. 5, 908-910 (1993).

[7] V. Baby, L. R. Chen, S. Doucet, and S. LaRochelle, "Continuous-wave operation of semiconductor optical amplifier-based multiwavelength tunable fiber lasers with 25-GHz spacing", IEEE J. Sel. Top. Quantum Electron. 13, 764-769 (2007).

[8] A. Bellemare, M. Karasek, M. Rochette, S. LaRochelle, and M. Tetu, "Room temperature multifrequency erbiumdoped fiber lasers anchored on the ITU frequency grid", J. Lightwave Technol. 18, 825-831 (2000).

[9] H. S. Djie, B. S. Ooi, X. -M. Fang, Y. Wu, J. M. Fastenau, W. K. Liu, and M. Hopkinson, "Room temperature broadband emission of InGaAs/GaAs quantum-dots laser", Opt. Lett. 32, 44-46 (2007).

[10] C. L. Tan, H. S. Djie, Y. Wang, C. E. Dimas, V. Hongpinyo, Y. H. Ding, and B. S. Ooi, "Wavelength tuning and emission width widening of ultrabroad quantum dash interband laser," Appl. Phys. Lett. 93, 111101 1-3, (2008).

[11] A. Kovsh, I. Krestnikov, D. Livshits, S. Mikhrin, J. Weimert, and A. Zhukov, "Quamtum dot laser with $75 \mathrm{~nm}$ broad spectrum of emission", Opt Lett. 32, 793-795 (2007).

[12] C. Gmachl, D. L. Sivco, R. Colombelli, F. Capasso, and A. Y. Cho, "Ultra-broadband semiconductor laser", Nature 415, 883-887 (2002).

[13] G. Sek, P. Podemski, A. Musial, J. Misiewicz, S. Hein, S. Hofling, and A. Forchel, "Exciton and biexciton emission from a single InAs/InP quantum dash", J. Appl. Phys. 105, 086104 (2009).

${ }^{[14]}$ R. H. Wang, A. Stintz, P. M. Varangis, T. C. Newell, H. Li, K. J. Malloy, and L. F. Lester, "Room-temperature operation of InAs quantum-dash lasers on InP (001)," IEEE Photon. Technol. Lett. 13, 767-769, (2001).

[15] S. Deubert, A. Somers, W. Kaiser, R. Schwertberger, J. P. Reithmaier. And A. Forchel, "InP-based quantum dash lasers for wide gain bandwidth applications", J. Crystal Growth 278, 346 (2005).

[16] C. L. Tan, H. S. Djie, Y. Wang, C. E. Dimas, V. Hongpinyo, Y. H. Ding, and B. S. Ooi, "The influence of nonequilibrium distribution on room temperature lasing spectra in quantum dash lasers", IEEE Photon. Technol. Lett. 21, 30-32 (2009).

[17] A. Markus, J. X. Chen, C. Paranthoen, A. Fiore, C. Platz, and O. Gauthier-Lafaye, "Simultaneous two-state lasing in quantum-dot lasers," Appl. Phys. Lett. 82, 1818-1820, (2003).

${ }^{[18]}$ Z. -C. Lin, C. -Y. Lu, and C. -P. Lee, "Self-assembled InAs quantum wire lasers", Semicond. Sci. Technol. 21, 1221-1223 (2006).

[19] M. Grundmann and D. Bimberg, "Gain and threshold of quantum dot lasers: theory and comparison to experiments”, Jpn. J. Appl. Phys. 36, 4181-4187 (1997).

${ }^{[20]}$ Q. Zhu, K. F. Karlsson, E. Pelucchi, and E. Kapon, "Transition from two-dimensional to three-dimensional quantum confinement in semiconductor quantum wires/quantum dots", Nano Lett. 7, 2227-2233 (2007).

[21] T. Otterburg, D. Y. Oberli, M. A. Dupertuis, N. Moret, E. Pelucchi, B. Dwir, K. Leifer, E. Kapon, "Enhancement of the binding energy of charged excitons in disordered quantum wires", Phys. Rev. B 71, 033301 (2005).

[22] A. Tredicucci, C. Gmachl, F. Capasso, D. L. Sivco, A. L. Hutchinson, and A. Y. Cho, "A multiwavelength semiconductor laser", Nature 396, 350-353 (1998). 